\title{
Microwave-Assisted Organocatalysis: Phosphine-Mediated Tomita Zipper Cyclization Affording Functionalized Spirooxindole
}

\author{
Xiao-Yan Zhang, ${ }^{\text {a,b }}$ Ze Shen, ${ }^{\mathrm{a}} \mathrm{Li}-\mathrm{Li} \mathrm{Hu},{ }^{\mathrm{a}}$ Liang-Jun Wang, ${ }^{\mathrm{a}}$ You-Shuai Lin, ${ }^{\mathrm{a}} \mathrm{Jian}-\mathrm{Wu} \mathrm{Xie}{ }^{\mathrm{b}, *}$ and Hai-Lei Cui ${ }^{\mathrm{a}, *}$ \\ ${ }^{a}$ International Academy of Targeted Therapeutics and Innovation, Chongqing University of Arts and Sciences, 319 Honghe Ave., Yongchuan,
}

Chongqing 402160, P.R. China; E-mail: cuihailei616@163.com

${ }^{b}$ Key Laboratory of the Ministry of Education for Advanced Catalysis Materials, Department of Chemistry and Life Science, Zhejiang Normal

University, Jinhua 321004, P. R. China; E-mail: xiejw@zjnu.cn

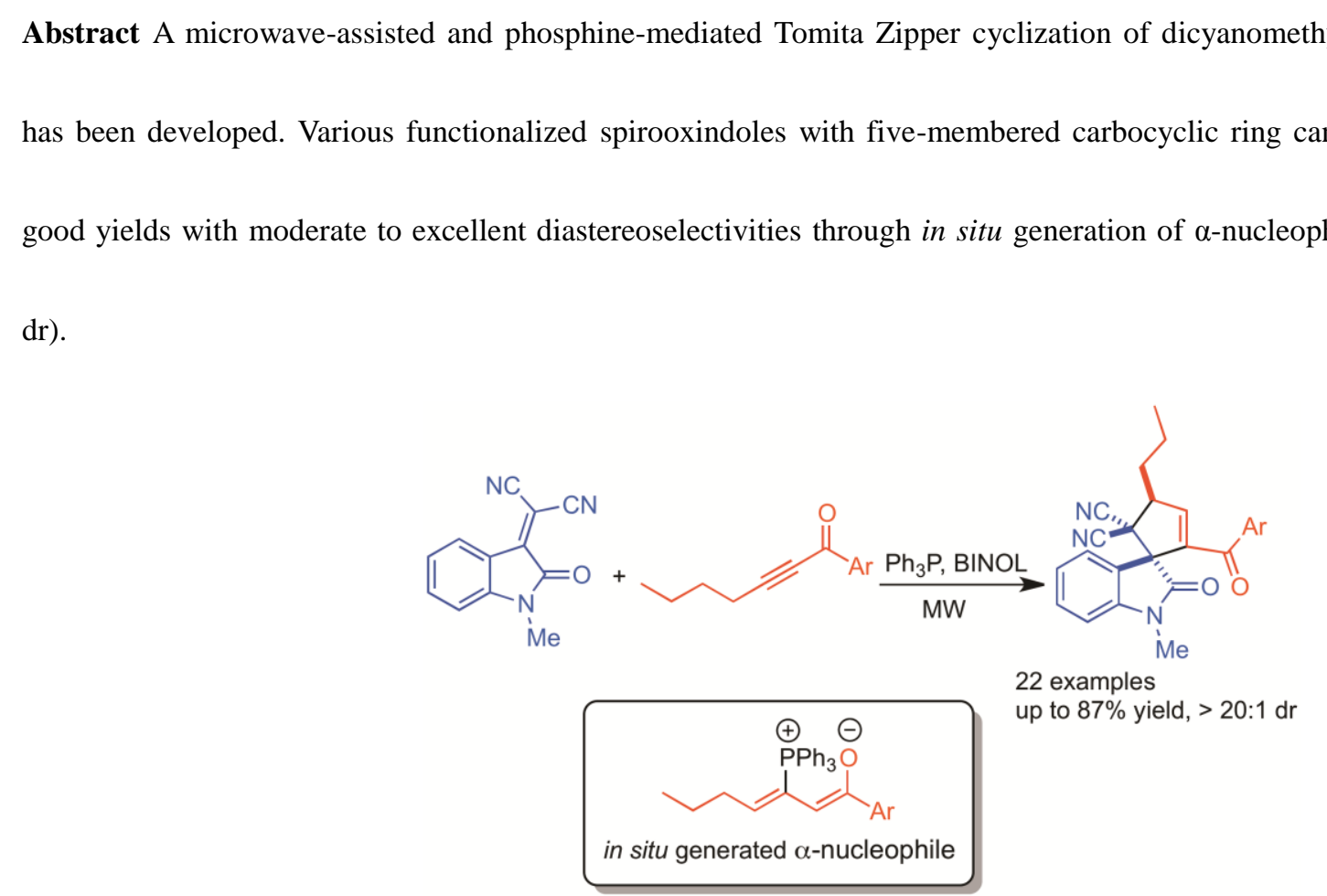

Keywords: Tomita Zipper cyclization, Microwave, Phosphine, Spirooxindole, Dicyanomethylideneoxindole

Spirooxindoles can be found in a large number of biologically active compounds and pharmaceuticals. Particularly, all-carbon five-membered spirooxindoles are featured in numerous natural products and synthetic bioactive compounds, such as Marcfortine B,

Spirotryprostatin B and Cyclopiamine B (Figure 1). ${ }^{1}$ Considering the intriguing molecular architecture and potent biological activities, various protocols have been developed to synthesize this class of molecules. ${ }^{2,3}$ However, the diastereoselective synthesis 
of five-membered spirooxindole, especially functionalized all-carbon spirocyclopenteneoxindole containing two quaternary carbons, still remains a challenging task.

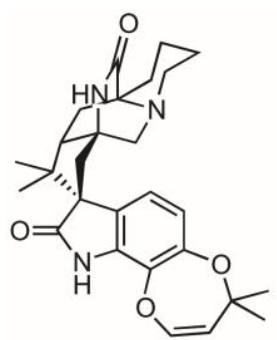

Marcfortine B

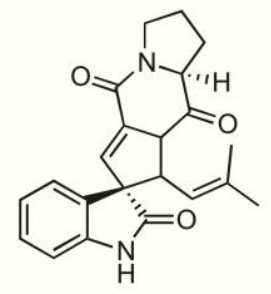

Spirotryprostatin B

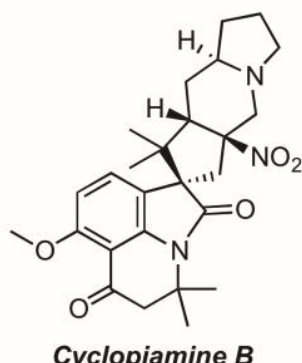

Cyclopiamine B

Figure 1. Natural products bearing all-carbon five-membered spirooxindole.

Recently, organocatalytic transformation of electron-deficient triple bonds has been emerging as a powerful method for the construction of carbocycles and heterocycles. ${ }^{4}$ For instance, Tomita group ${ }^{5}$ and $\mathrm{Fu}$ group ${ }^{6}$ reported their strategies of phosphine-catalyzed synthesis of bicycles with alkynyl ketones. A novel $\alpha^{\prime}$-nucleophile, (Z)-4-(tributylphosphonio)buta-1,3-dien-2-olate, was formed in these intramolecular reactions. Ramachary and co-workers disclosed a phosphine-catalyzed stereoselective synthesis of functionalized five-membered spirooxindole through intermolecular Tomita Zipper Cyclization. ${ }^{7}$ Xue group have also employed tributylphosphine for activation of alkynyl ketone to generate a nucleophile at the $\alpha$-position. The in situ generated $\alpha$-nucleophile could react with $N$-Tosylimines delivering pyrrolidines in good to excellent yields. ${ }^{8}$ Inspired by these pioneering achievements of phosphine-catalyzed reactions by the groups of Tomita ${ }^{5}, \mathrm{Fu}^{6}, \mathrm{Lu}^{9}{ }^{9}$ Shi, ${ }^{10}$ Ramachary, ${ }^{7}$ Kwon ${ }^{11}$ and others, ${ }^{12,13}$ we hypothesized that phosphine-catalyzed transformation of alkynyl ketones could be applied in the construction of all-carbon five-membered spirooxindoles via a Tomita Zipper cyclization (Scheme 1). ${ }^{14}$ $\alpha$-Nucleophile would be generated when $\alpha^{\prime}$-blocked ynone is attacked by a nucleophilic catalyst and the subsequent tandem reactions would allow access to spirocyclopenteneoxindole. ${ }^{15}$ Herein, we report our development of a microwave-assisted and phosphine-mediated synthesis of spirooxindoles with five-membered carbocyclic ring. 
Ramachary, et al: $\alpha$ '-nucleophile formation

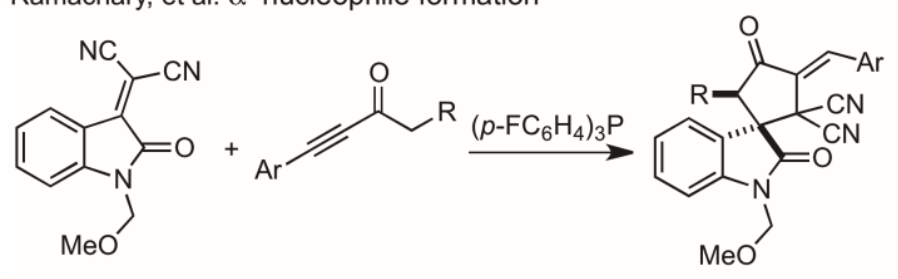

this work: $\alpha$-nucleophile formation

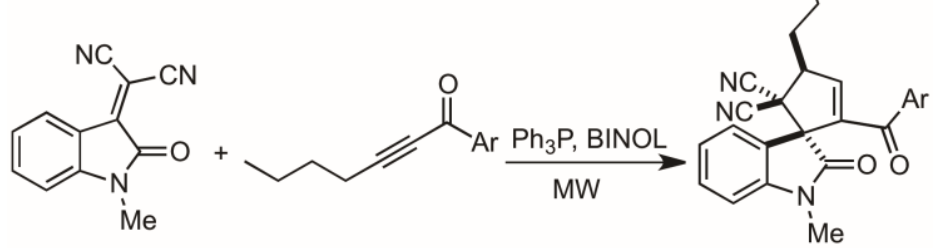<smiles>[R]CC(=O)C#C[Bi]</smiles>

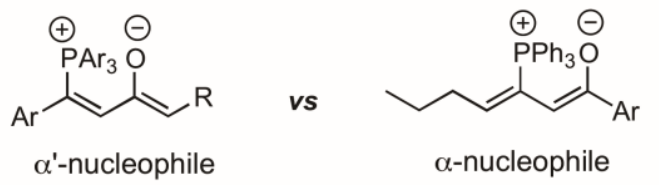

Scheme 1. Design for the synthesis of all-carbon five-membered spirooxindoles.

We began our study with olefin 1a and ynone $2 \mathbf{a}$ as model substrates in toluene to react with each other in the presence of 30 mol\% of $\mathrm{Ph}_{3} \mathrm{P}$ under irradiation of microwave. ${ }^{16}$ Pleasingly, the desired all-carbon five-membered spirooxindole 3a could be detected with a promising $24 \%$ NMR yield (Table 1, entry 1). With $30 \mathrm{~mol} \%$ of BINOL as additive, an improved $40 \%$ yield could be observed (entry 2). Addition of $4 \AA$ MS had no significant influence on yield (entry 3). Increasement of catalyst loading led to a slightly improved yield (entry 4). Higher reaction temperature gave decreased yield, while lowering temperature afforded increased yield (entries 5-7). Improvement of yield was obtained when the reaction was performed with an excess amount of ynone (entry 8). However, the absence of BINOL resulted in a dramatically decreased yield (entry 9). We reasoned that the role of BINOL in this system is to activate ynone, stabilize enolate intermediate by hydrogen bonding and facilitate proton transfer. ${ }^{17,18,19}$ The raction by conventioanl heating gave lower yield and was not as clean as that by microwave heating, showing the advantages of microwave energy for this reaction (entry 10).

Table 1 
Selected optimization studies ${ }^{\mathrm{a}, \mathrm{b}, \mathrm{c}}$

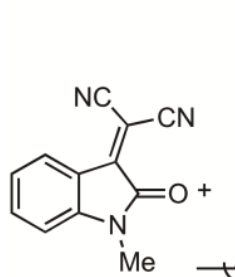<smiles>CCC[C@H]1C=C(C(=O)c2ccc(OC)cc2)[C@@]2(C(=O)N(C)c3ccc(C(C)C)cc32)[C@@]1(C)C#N</smiles>

$2 a$

\begin{tabular}{lllll}
\hline Entry & Additive & $\begin{array}{l}\text { Ratio } \\
(\mathbf{1 a : 2 a : P h} \text { P:Additive })\end{array}$ & $\mathrm{T}\left({ }^{\circ} \mathrm{C}\right)$ & Yield [\%] \\
\hline 1 & - & $1: 1.2: 0.3: 0$ & 150 & 24 \\
2 & BINOL & $1: 1.2: 0.3: 0.3$ & 150 & 40 \\
$3^{d}$ & BINOL & $1: 1.2: 0.3: 0.3$ & 150 & 38 \\
4 & BINOL & $1: 1.2: 0.6: 0.3$ & 150 & 43 \\
5 & BINOL & $1: 1.2: 0.6: 0.3$ & 180 & 26 \\
6 & BINOL & $1: 1.2: 0.6: 0.3$ & 100 & 63 \\
7 & BINOL & $1: 1.2: 0.6: 0.3$ & 80 & 59 \\
8 & BINOL & $1: 2: 0.6: 0.3$ & 100 & $79(71)^{\mathrm{e}}$ \\
9 & - & $1: 2: 0.6: 0$ & 100 & 25 \\
$10^{\dagger}$ & BINOL & $1: 2: 0.6: 0.3$ & 100 & 61 \\
\hline
\end{tabular}

${ }^{\text {a }}$ Unless otherwise noted, reaction was performed with $0.2 \mathrm{mmol}$ of $\mathbf{1 a}, 0.24 \mathrm{mmol}$ of $\mathbf{2 a}$ and 30 mol\% of catalyst in $1.0 \mathrm{~mL}$ of toluene irradiated by microwave for 20 minutes.

${ }^{\text {b }}$ Yield was determined by ${ }^{1} \mathrm{H}$ NMR with $\mathrm{CH}_{2} \mathrm{Br}_{2}$ as internal standard.

${ }^{\mathrm{c}}$ Excellent diastereoselectivities (>20:1) was observed in all cases.

${ }^{\mathrm{d}}$ With $100 \mathrm{mg}$ of $4 \AA \mathrm{MS}$.

e Isolated yield.

${ }^{\mathrm{f}}$ Heated at $100{ }^{\circ} \mathrm{C}$ for 20 minutes by conventional heating.

With optimized conditions in hand, we then examined the substrate scope of olefins. As shown in Table 2, dicyanomethylideneoxindoles bearing methyl, methoxymethyl, propargyl, benzyl and phenyl groups at N1 position can be tolerated in this process, delivering corresponding spirocyclopenteneoxindoles in moderate to good yields (61-81\%) and excellent diastereoselectivities (>20:1 in all cases). Lower catalyst loading and temperature can also afford desired products at the cost of prolonged reaction time when more reactive substrates were used (3f and $\mathbf{3 g}$ ). A drop in diastereoselectivity was observed in the cases of 4-substituted dicyanomethylideneoxindoles ( $\mathbf{3 h}$ and $\mathbf{3 i}, 3.3: 1-2.6: 1 \mathrm{dr})$. Dicyanomethylideneoxindoles $\mathbf{1}$ with electron-withdrawing groups and electron-donating groups at 5 and 6 positions gave functionalized five-membered spirooxindoles in moderate to good yields $(51-87 \%)$ with moderate to excellent diastereoselectivities $(3.3: 1->20: 1)$. Dicyano substituents were essential for this transformation, as methylideneoxindoles without dicyano substituents failed to deliver corresponding 
products. ${ }^{20}$ The stereochemistry and structure of oxindole spirocyclopentenes $\mathbf{3}$ were confirmed by X-ray structure analysis on compound $3 \mathbf{n} .^{21}$

\section{Table 2}

Examination of Substrate Scope $e^{\mathrm{a}, \mathrm{b}}$

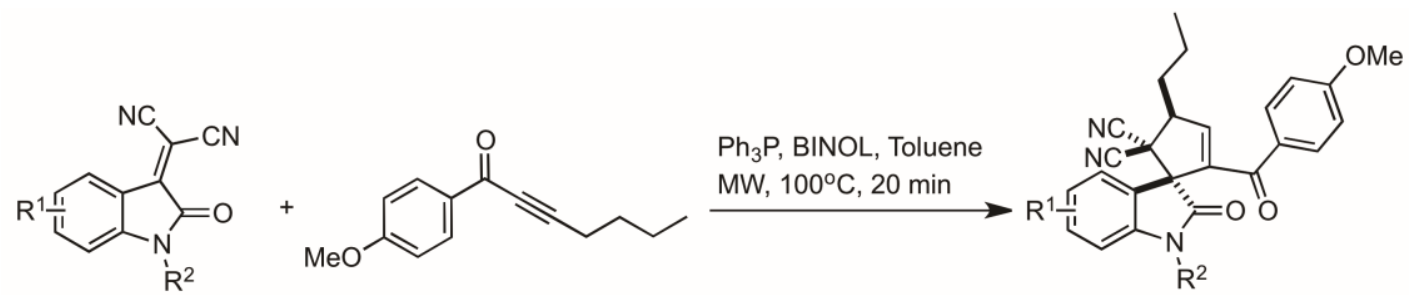<smiles>CCC[C@H]1C=C(C(=O)c2ccc(O)cc2)[C@@]2(C(=O)N(C)c3ccccc32)[C@@]1(C)C#N</smiles>

3a $71 \%,>20: 1 d r$

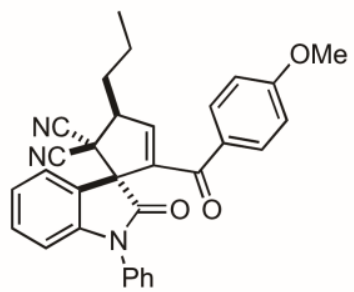

$3 e^{c} 77 \%,>20: 1 d r$<smiles>CCC[C@H]1C=C(C(=O)c2ccc(OC)cc2)[C@@]2(C(=O)N(C)c3cccc(Cl)c32)[C@H]1C#N</smiles>

3i $66 \%, 3.3: 1 \mathrm{dr}$

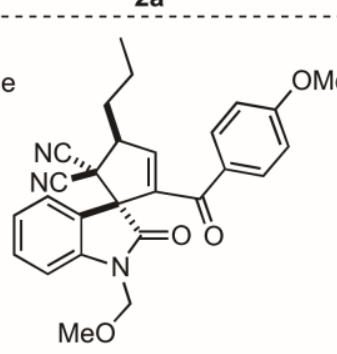

3b $61 \%,>20: 1 \mathrm{dr}$

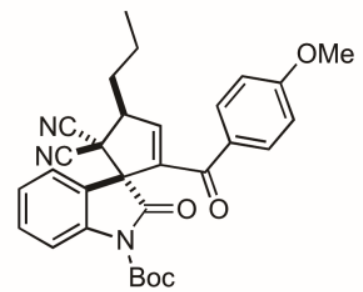

$3 f^{d} 78 \%,>20: 1 d r$<smiles>C#CCN1C(=O)C2(C(CCC)C=CC2C(=O)c2ccc(OC)cc2)[C@H](C#N)C1C#N</smiles>

3c $81 \%,>20: 1 \mathrm{dr}$<smiles>CCC[C@H]1C=C(C(=O)c2ccc(OC)cc2)[C@@]2(C(=O)N(C)c3ccccc32)C1(C#N)C#N</smiles>

$3 g^{e} 73 \%$, > 20:1 dr
$3 \mathrm{a}$<smiles>CCC[C@@H]1C=C(C(=O)c2ccc(OC)cc2)[C@@]2(C(=O)N(Cc3ccccc3)c3ccccc32)C1(C#N)C#N</smiles>

3d $62 \%$, > 20:1 dr

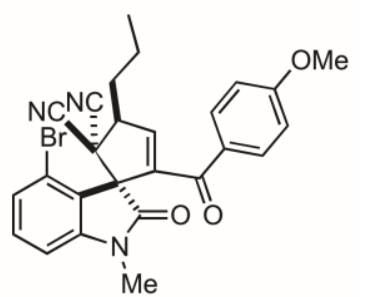

3h $59 \%, 2.6: 1 \mathrm{dr}$<smiles>CCC[C@H]1C=C(C(=O)c2ccc(OC)cc2)[C@@]2(C(=O)N(C)c3ccc(C)cc32)C1=C[C@H](CCC)[C@@]1(C#N)C(=O)N(C)c2cc(Br)ccc21</smiles>

$3 \mathrm{~m} \mathrm{76 \% ,} \mathrm{3.3:1} \mathrm{dr}$<smiles>CCC[C@H]1C=C(C(=O)c2ccc(OC)cc2)[C@@]2(C(=O)N(C)c3ccc(Br)cc32)C1=C[C@H](CCC)[C@@]1(C#N)C(=O)N(C)c2ccc([N+](=O)[O-])cc2[C@]12C(=O)N(C)c1ccc(Cl)cc12</smiles>

3k $87 \%$, > 20:1 dr

3I $58 \%,>20: 1 \mathrm{dr}$

3n $80 \%$, > 20:1 dr

${ }^{\text {a }}$ Unless otherwise noted, reaction was performed with $0.2 \mathrm{mmol}$ of $\mathbf{1}, 0.4 \mathrm{mmol}$ of $\mathbf{2}, 60 \mathrm{~mol} \% \mathrm{of} \mathrm{PPh}_{3}$ and $30 \mathrm{~mol} \%$ of $\mathrm{BINOL}$ in $1.0 \mathrm{~mL}$ of toluene under microwave irradiation at $100{ }^{\circ} \mathrm{C}$ for 20 minutes.

b Isolated yields. 
${ }^{\mathrm{c}}$ Performed at $100{ }^{\circ} \mathrm{C}$ for 25 minutes.

${ }^{\text {d }}$ Performed with $30 \mathrm{~mol} \%$ of $\mathrm{PPh}_{3}$ and $10 \mathrm{~mol} \%$ of BINOL at $70{ }^{\circ} \mathrm{C}$ for 90 minutes.

${ }^{\text {e }}$ Performed with $30 \mathrm{~mol} \%$ of $\mathrm{PPh}_{3}$ and $10 \mathrm{~mol} \%$ of BINOL at $70{ }^{\circ} \mathrm{C}$ for 120 minutes.

Next, we explored the scope of alkynyl ketones under this catalytic system. As shown in Table 3, various aryl alkynyl ketones with phenyl ring or electron-deficient and electron-rich substituents on the phenyl ring delivered desired five-membered spirooxindoles in moderate to good yields (47-82\%). Heteroaryl substituted ynone can also be applied in this process giving $65 \%$ yield $(\mathbf{3 u})$. Unfortunately, this method has its limitations. Corresponding product $\mathbf{3 v}$ could not be obtained under the current system in the case of alkylated alkynyl ketone. Product 3w could be obtained when ynone bearing longer chain was employed in this reaction, albeit with decreased yield. Ynone with phenyl group at $\psi$-position failed to deliver spirocyclopenteneoxindole $3 \mathbf{x}$ too. ${ }^{22}$ Complex mixture were observed instead of spirocyclopentene-oxindoles in the two cases of $\mathbf{3 v}$ and $\mathbf{3 x}$.

\section{Table 3}

Examination of Substrate Scope $\mathrm{a}^{\mathrm{a}, \mathrm{b}}$ 


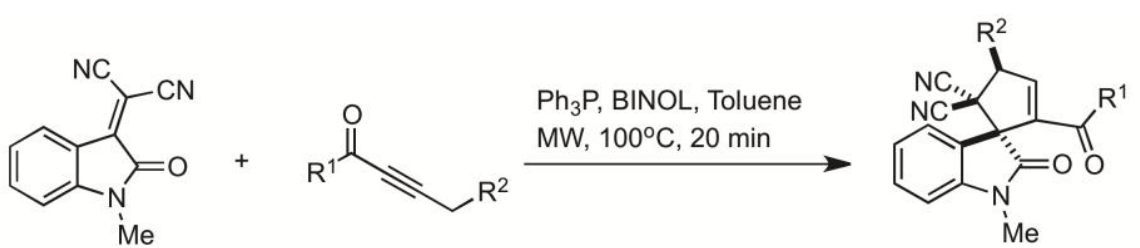

$1 \mathrm{a}$ 2

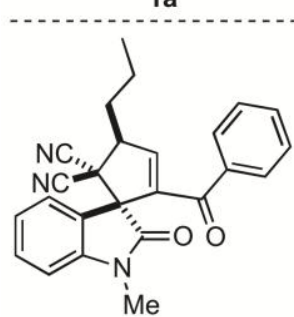

3o $79 \%, 6.3: 1 \mathrm{dr}$<smiles>CCC[C@@H]1C=C(C(=O)c2ccc(C)cc2)[C@@]2(C(=O)N(C)c3cccnc32)C1(C#N)C#N</smiles>

3s $67 \%$, > 20:1 dr

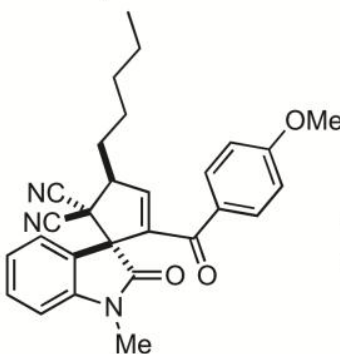

$\mathrm{Me}$

$3 w 44 \%,>20: 1 d r$

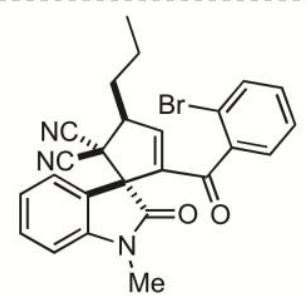

3p $47 \%, 3.2: 1 \mathrm{dr}$

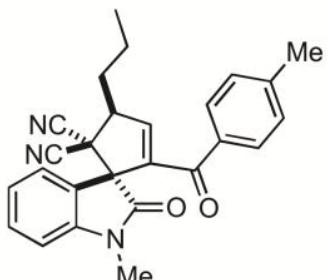

3t $67 \%, 7.3: 1 \mathrm{dr}$

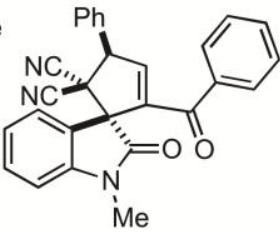

$3 x^{c}$ ND

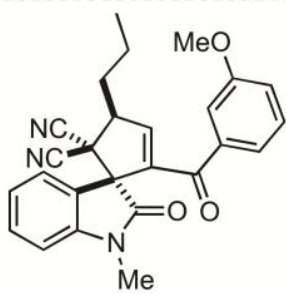

$3 q 82 \%,>20: 1 \mathrm{dr}$

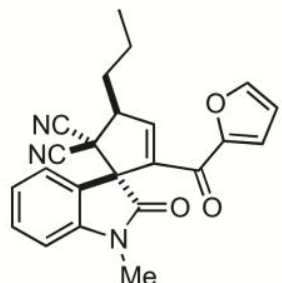

3u $65 \%$, > 20:1 dr

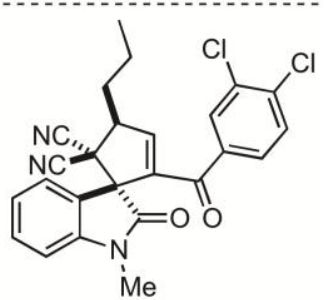

$3 \mathrm{r} 62 \%, 9.0: 1 \mathrm{dr}$<smiles>CCC[C@H]1C=C(C(=O)C2CCCCC2)[C@@]2(C(=O)N(C)c3ccccc32)C1(C#N)C#N</smiles>

$3 v^{c}$ ND

${ }^{a}$ Unless otherwise noted, reaction was performed with $0.2 \mathrm{mmol}$ of $\mathbf{1}, 0.4 \mathrm{mmol}$ of $\mathbf{2}, 60 \mathrm{~mol} \% \mathrm{of} \mathrm{PPh}_{3}$ and $30 \mathrm{~mol} \%$ of BINOL in $1.0 \mathrm{~mL}$ of toluene under microwave irradiation at $100{ }^{\circ} \mathrm{C}$ for 20 minutes.

${ }^{\mathrm{b}}$ Isolated yields.

${ }^{\mathrm{c}}$ Not detected.

We proposed a possible mechanism for this reaction on the basis of our results and previous reports from other groups (Scheme 2). ${ }^{17,18}$ Attack of phosphine to hydrogen bonding activated ynone gives a zwitterionic intermediate $\mathbf{A}$ which would be subsequently transformed to intermediate $\mathbf{B}$ via intramolecular proton-transfer. $\alpha$-Nucleophile $\mathbf{D}$ as a reactive resonance contributing structure undergoes Michael reaction giving carbanion species E. Further intramolecular addition, proton migration and elimination deliver the desired oxindole spirocyclopentene $\mathbf{3}$. As intermediates $\mathbf{C}$ and $\mathbf{D}$ are more stable than intermediate $\mathbf{B}$, $\alpha$-addition is favored leading to excellent regioselectivities in all cases. BINOL as a hydrogen-bonding donor in this system can activate ynone 2, stabilize enolate $\mathbf{D}$ and be beneficial for proton transformation. This may explain the improvement of yield by adding BINOL to reaction system. 


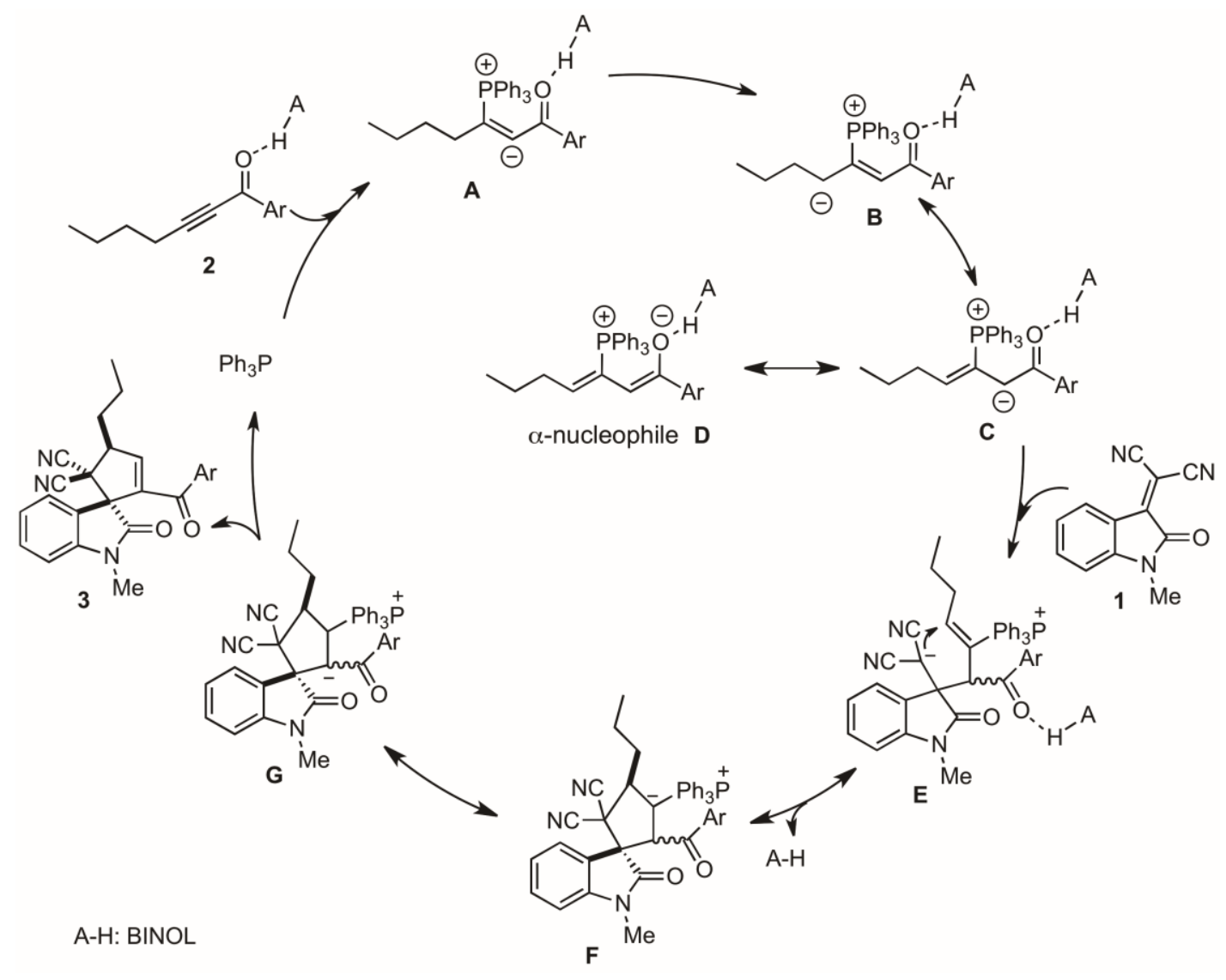

Scheme 2. Proposed Catalytic Cycle

In conclusion, we have developed a microwave-assisted and phosphine-mediated construction of spirocyclopentene-oxindoles from simple dicyanomethylideneoxindoles and ynones. The reactions proceeded through a Tomita Zipper cyclization involving the in situ generation of $\alpha$-nucleophile. Various functionalized spirooxindoles with five-membered carbocyclic ring can be obtained in moderate to good yields with moderate to excellent diastereoselectivities (up to $87 \%$ yield, > 20:1 dr). Application of this methodology in biomedical research is underway in our laboratory.

\section{Acknowledgments}

We are grateful for the support provided for this study by the National Science Foundation of China (21502013, 21272214), the Scientific and Technological Research Program of the Chongqing Municipal Education Commission (KJ1501111), and Chongqing University of Arts and Sciences (R2015BX01). 


\section{References and notes}

1. (a) Galliford, C. V.; Scheidt, K. A. Angew. Chem., Int. Ed., 2007, 46, 8748; (b) Trost, B. M.; Brennan, M. K. Synthesis, 2009, 3003; (c) Trost, B. M.; Cramer, N.; Bernsmann, H. J. Am. Chem. Soc., 2007, 129, 3086; (d) Fensome, A.; Adams, W. R.; Adams, A.

L.; Berrodin, T. J.; Cohen, J.; Huselton, C.; Illenberger, A.; Kern, J. C.; Hudak, V. A.; Marella, M. A.; Melenski, E. G.; McComas, C. C.; Mugford, C. A.; Slayden, O. D.; Yudt, M.; Zhang, Z.; Zhang, P.; Zhu, Y.; Winneker, R. C.; Wrobel, J. E. J. Med. Chem., 2008, 51, 1861; (e) Antonchick, A. P.; Gerding-Reimers, C.; Catarinella, M.; Schürmann, M.; Preut, H.; Ziegler, S.; Rauh, D.; Waldmann, H. Nat. Chem., 2010, 2, 735; (f) Mercado-Marin, E. V.; Garcia-Reynaga, P.; Romminger, S.; Pimenta, E. F.; Romney, D. K.; Lodewyk, M. W.; Williams, D. E.; Andersen, R. J.; Miller, S. J.; Tantillo, D. J.; Berlinck, R. G. S.; Sarpong, R. Nature., 2014, 509, 318; (g) Li, S.-M. Nat. Prod. Rep., 2010, 27, 57.

2. For reviews, see: (a) Liang, H.; Wang, R. Adv. Synth. Catal., 2013, 355, 1023; (b) Singh, G. S.; Desta, Z. Y. Chem. Rev., 2012, 112, 6104; (c) Dalpozzo, R.; Bartoli, G.; Bencivenni, G. Chem. Soc. Rev., 2012, 41, 7247; (d) Ball-Jones, N. R.; Badillo, J. J.; Franz, A. K. Org. Biomol. Chem., 2012, 10, 5165; (e) Chauhan, P.; Chimni, S. S. Tetrahedron: Asymmetry, 2013, 24, 343; (f) Zhou, F.; Liu, Y.-L.; Zhou, J. Adv. Synth. Catal., 2010, 352, 1381; (g) Cheng, D.; Ishihara, Y.; Tan, B.; Barbas II C. F. ACS Catal., 2014, 4, 743; (h) Rios, R. Chem. Soc. Rev., 2012, 41, 1060; (i) Marti, C.; Carreira, E. M. Eur. J. Org. Chem., $2003,2209$.

3. For selected examples on the synthesis of all-carbon five-membered spirooxindoles, see: (a) Ball-Jones, N. R.; Badillo, J. J.; Tran, N. T.; Franz, A. K. Angew. Chem., Int. Ed., 2014, 53, 9462; (b) Sun, Q.-S.; Zhu, H.; Chen, Y.-J.; Yang, X.-D.; Sun, X.-W.; Lin, G.-Q. Angew. Chem., Int. Ed., 2015, 54, 13253; (c) Zhou, J.; Wang, Q.-L.; Peng, L.; Tian, F.; Xu, X.-Y.; Wang, L.-X. Chem. Commun., 2014, 50, 14601; (d) Sun, W.; Zhu, G.; Wu, C.; Hong, L.; Wang, R. Chem. Eur. J., 2012, 18, 6737; (e) Gomez, C.; Gicquel, M.; Carry, J.-C.; Schio, L.; Retailleau, P.; Voituriez, A.; Marinetti, A. J. Org. Chem., 2013, 78, 1488; (f) Trost, B. M.; Cramer, N.; Silverman, S. M. J. Am. Chem. Soc., 2007, 129, 12396; (g) Albertshofer, K.; Tan, B.; Barbas III C. F. Org. Lett., 2012, 14, 1834; (h) Jiang, K.; Tiwari, B.; Chi, Y. R. Org. Lett., 2012, 14, 2382; (i) Patra, A.; Bhunia, A. Yetra, S. R.; Gonnade, R. G.; Biju, A. T. Org. Chem. Front., 2015, 2, 1584; (j) Selvakumar, K.; Vaithiyanathan, V.; Shanmugam, P. Chem. Commun., 2010, 46, 2826. 
4. For a review, see: Fraile, A.; Parra, A.; Tortosa, M.; Alemán, J. Tetrahedron, 2014, 70, 9145.

5. Kuroda, H.; Tomita, I.; Endo, T. Org. Lett., 2003, 5, 129.

6. Wilson, J. E.; Sun, J.; Fu, G. C. Angew. Chem., Int. Ed., 2010, 49, 161.

7. Ramachary, D. B.; Venkaiah, C.; Krishna, P. M. Org. Lett., 2013, 15, 4714.

8. Meng, L.-G.; Cai, P.; Guo, Q.; Xue, S. J. Org. Chem., 2008, 73, 8491.

9. Lu, C.; Lu, X. Org. Lett., 2002, 4, 4677.

10. Zhang, X.-C.; Cao, S.-H.; Wei, Y.; Shi, M. Org. Lett., 2011, 13, 1142

11. Tran, Y. S.; Kwon, O. Org. Lett., 2005, 7, 4289.

12. (a) Han, X.; Yao, W.; Wang, T.; Tan, Y. R.; Yan, Z.; Kwiatkowski, J.; Lu, Y. Angew. Chem., Int. Ed., 2014, 53, 5643; (b) Gabillet, S.; Loreau, O.; Specklin, S.; Rasalofonjatovo, E.; Taran, F. J. Org. Chem., 2014, 79, 9894; (c) Gicquel, M.; Zhang, Y.; Aillard, P.; Retailleau, P.; Voituriez, A.; Marinetti, A. Angew. Chem., Int. Ed., 2015, 54, 5470; (d) Han, Y.; Sheng, Y.-J.; Yan, C.-G. Org. Lett., 2014, 16, 2654; (e) Hu, J.; Wei, Y.; Tong, X. Org. Lett., 2011, 13, 3068; (f) Liao, J.-Y.; Shao, P.-L.; Zhao, Y. J. Am. Chem. Soc., 2015, 137, 628; (g) Xu, S.; Zhou, L.; Zeng, S.; Ma, R.; Wang, Z.; He, Z. Org. Lett., 2009, 11, 3498; (h) Wang, T.; Ye, S. Org. Lett., 2010, 12, 4168.

13. For reviews on nucleophilic catalysis, see: (a) Methot, J. L.; Roush, W. R. Adv. Synth. Catal., 2004, 346, 1035; (b) Fan, Y. C.; Kown, O. Chem. Commun., 2013, 49, 11588; (b) Wei, Y.; Shi, M. Chem. Asian. J., 2014, 9, 2720; (c) Xiao, Y.; Sun, Z.; Guo, H.; Kwon, O. Beilstein J. Org. Chem., 2014, 10, 2089; (d) Voituriez, A.; Marinetti, A.; Gicquel, M. Synlett, 2015, $26,142$. 14. For selected examples on phosphine-catalyzed formal [3+2] cyclization through activation of $\alpha^{\prime}$-position of alkynyl ketone, see: (a) Ramachary, D. B.; Venkaiah, C.; Krishna, P. M. Chem. Commun., 2012, 48, 2252; (b) Liang, L.; Li, E.; Xie, P.; Huang, Y. Chem. Asian. J., 2014, 9, 1270; (c) Lian, Z.; Shi, M. Eur. J. Org. Chem., 2012, 581; (d) Lian, Z.; Shi, M. Org. Biomol. Chem., 2012, 10, 8048; (e) Yang, L.; Xie, P.; Li, E.; Li, X.; Huang, Y.; Chen, R. Org. Biomol. Chem., 2012, 10, 7628; (f) Zhou, Q.-F.; Chu, X.-P.; Ge, F.-F.; Li, C.; Lu, T. Mol. Diversity, 2013, 17, 563. For selected examples on synthesis of five-membered spirooxindole 
with allenoates and dicyanomethylideneoxindoles, see: (g) Guo, S.; Wang, R.; Li, J.: Li, C.; Deng, H.; Jia, X. Synlett, 2011, 15, 2256; (h) Li, J.; Wang, N.; Li, C.; Jia, X. Chem. Eur. J., 2012, 18, 9650.

15. Generation of $\alpha$-nucleophile from ynone under the catalysis of phosphine is uncommen as $\mathrm{PPh}_{3}$ can also promote isomerization of yne-carbonyl compounds to conjugated diene carbonyl compounds at $80{ }^{\circ} \mathrm{C}$ in good yield, see: (a) Trost, B. M.; Kazmaier, U. $J$. Am. Chem. Soc., 1992, 114, 7933; (b) Ma, D.; Yu, Y.; Lu, X. J. Org. Chem., 1989, 54, 1105.

16. For reviews, see: (a) Larhed, M.; Moberg, C.; Hallberg, A. Acc. Chem. Res., 2002, 35, 717; (b) Roberts, B.; Strauss, C. R. Acc. Chem. Res., 2005, 38, 653; (c) Lew, A.; Krutzik, P. O.; Hart, M. E.; Chamberlin, A. R. J. Comb. Chem., 2002, 4, 95; (d) Lidström, P.; Tierney, J.; Wathey, B.; Westman, J. Tetrahedron, 2001, 57, 9225; (e) Perreux, L.; Loupy, A. Tetrahedron, 2001, 57, 9199. For the microwave assisted phosphine-organocatalysis, see: Lopes, S. M. M.; Santos, B. S.; Palacios, F.; Melo, T. Arkivoc, $2010,78$.

17. For reviews, see: (a) Doyle, A. G.; Jacobsen, E. N. Chem. Rev., 2007, 107, 5713; (b) Takemoto, Y. Org. Biomol. Chem., 2005, 3, 4299; (c) Connon, S. J. Chem. Eur. J., 2006, 12, 5418; (d) Akiyama, T. Chem. Rev., 2007, 107, 5744.

18. For selected examples on combination of nucleophilic catalysis and hydrogenbonding catalysis, see: (a) van Steenis, D. J. V. C. Marcelli, T.; Lutz, M.; Spek, A. L.; van Maarseveen, J. H.; Hiemstra, H. Adv. Synth. Catal. 2007, 349, 281; (b) Jiang, Y.-Q.; Shi, Y.-L.; Shi, M. J. Am. Chem. Soc. 2008, 130, 7202; (c) Cui, H.-L.; Peng, J.; Feng, X.; Du, W.; Jiang, K.; Chen, Y.-C. Chem.-Eur. J. 2009, 15, 1574.

19. For other optimaziton results, see supporting information.

20. See supporting information.

21. CCDC 1449553 for compound 3n. See supporting information for details.

22. Treatment of propargylic ester and dicyanomethylidene-oxindole under this catalytic system led to no reaction. See supporting information. 